\title{
Intercambio de experiencias de capacitación y de práctica profesional
}

\author{
Marielos Vargas Morales ${ }^{1}$ \\ Académica de la División de Educación Rural del \\ Centro de Investigación y Docencia en Educación (CIDE), Universidad Nacional \\ Heredia, Costa Rica
}

Recibido 11 de agosto de 2009• Aceptado 02 de diciembre de 2009

Resumen. Este artículo surge como producto de la implementación del proyecto Intercambio Académico entre la División de Educación Rural (DER), de la Universidad Nacional (UNA) y escuelas colaboradoras. Desde su quehacer se abordan dos acciones fundamentales; la primera, la concepción teórico-metodológica de la práctica profesional de la DER y la segunda, el proceso de capacitación dirigido a maestros rurales en servicio. A partir de estas actividades académicas se exponen productos gestados a lo interno del proyecto y algunas reflexiones sobre la experiencia académica como resultado de lecciones aprendidas durante el proceso.

Palabras clave. Educación rural, formación inicial, práctica profesional, educación continua.

Abstract. The present article arises from the implementation of the Academic Exchange project between the Division de Educación Rural (Rural Education School) from Universidad Nacional and other collaborating schools. There are two fundamental actions that are approached; first, the theoretical methodological conception of the professional field practicum of the DER and second, the teaching training to rural teachers in service. From these academic activities, products developed are exposed in the project and some reflections about the academic experience were also exposed as a result of lessons learned during the whole process.

Key words. Rural education, initial formation, professional field practicum, continuous education.

${ }^{1}$ Licenciada en Educación con énfasis en Educación Rural de I y II ciclos por la Universidad Nacional de Costa Rica. Se ha desempeñado como docente en escuelas rurales y urbanas del país. Es académica y extensionista de la División de Educación Rural del Centro de Investigación y Docencia en Educación (CIDE), de la Universidad Nacional (UNA), Costa Rica.

Correo electrónico: maryvamo39@gmail.com

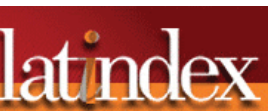

La Revista Electrónic@ Educare está indizada en el Catálogo LATINDEX. 


\section{Introducción}

El intercambio académico entre las escuelas colaboradoras ${ }^{2}$ y la División de Educación Rural (DER) es un proyecto que surgió ante la necesidad de contribuir con el mejoramiento de la calidad educativa de las escuelas rurales, en congruencia con el plan de estudios de la DER, cuyo quehacer se ha enfocado en la formación y la capacitación de los profesionales que se desempeñan en diferentes contextos educativos rurales de nuestro país, así como la necesidad de articular los procesos de inserción de los estudiantes de la práctica profesional en un contexto escolar rural.

El intercambio académico se inició en 2006 y finalizó en 2008, a partir de un plan de capacitación y actualización dirigido a aquellos docentes de la Educación General Básica de I y II ciclos, de la Dirección Regional de Enseñanza de Heredia, que aceptan estudiantes practicantes para el desarrollo de la práctica profesional propuesta por la División de Educación Rural, en los procesos de formación inicial docente, complementado con el desarrollo del proceso de práctica profesional de la DER.

Este artículo surge como resultado de la experiencia docente gestada en el marco del proyecto Intercambio Académico y no, simplemente, como producto de una investigación. Su objetivo es dar a conocer actividades y esfuerzos que se desarrollan en el seno de las unidades académicas, así como, también, dejar evidencia de las relaciones y los vínculos que se promueven en y desde la Universidad Nacional (UNA) con instituciones educativas en el ámbito nacional, con el propósito de contribuir al mejoramiento de la educación y, en este caso, en particular, a la educación que se desarrolla en contextos rurales.

\section{Consideraciones teóricas}

En la actualidad, la formación docente es una tarea desarrollada por un sinnúmero de instituciones tanto públicas como privadas, que proveen al país y al mayor ente empleador, el Ministerio de Educación Pública (MEP), de los profesionales orientados a la Educación General Básica (I y II ciclos). Desde el surgimiento de las primeras escuelas a lo largo y ancho de nuestro país, se ha dado una marcada diferencia en el acceso, las posibilidades y las oportunidades educativas en las distintas zonas rurales y urbanas; este desfase apunta a la necesidad de esfuerzos que contribuyan a la reducción de brechas que, con tendencia natural, se ensanchan, cada vez más, entre el campo y la ciudad.

Con base en un acercamiento a la pedagogía rural, Aguilar (1994) plantea la disparidad que existe entre la educación de las zonas rurales y las urbanas, y demuestra que se ha pensado en las realidades urbanas y se ha ignorado las particularidades de la sociedad rural, lo que implica que se carezca de soluciones para atender las necesidades relacionadas con el contexto y los intereses de los habitantes del medio rural.

La educación como factor de desarrollo, debe contribuir a reducir las brechas sociales existentes, por lo que el esfuerzo que se lleva a cabo para capacitar a los formadores en el área de la docencia debe constituirse en un elemento clave para potenciar las habilidades y las capacidades, no sólo de los docentes en formación, sino que trascienda la propia y se encauce el accionar hacia un impacto escolar y comunal en todos sus niveles.

${ }^{2}$ Escuelas colaboradoras son aquellas que brindan su institución como un espacio para el crecimiento pedagógico de los estudiantes practicantes, al permitirles realizar la práctica profesional en su instancia escolar. 
Se ha llegado a comprobar que algunos de los problemas inherentes al bajo rendimiento académico del sistema educativo, en la zona rural, resultan de aspectos que tienen relación con el origen, la formación y las características personales del profesor encargado (Gajardo y De Andraca, 1992).

Ante esta premisa, la formación docente no debe centrarse en la obtención de un título y dejar que éste se añeje en el recuerdo. Se requiere tener presentes las dinámicas nacional e internacional, especialmente esta última en la que la vida cotidiana evoluciona a pasos agigantados, y en la que el cambio, la incertidumbre, la diversidad, la inequidad, marcan la realidad de los diferentes escenarios de la sociedad; por esto, la capacitación continua debe ser una constante en la profesión docente, especialmente en aquellos que trabajan en contextos educativos socialmente frágiles.

Al respecto, Román (1997, citado por Aguilar y Solano, 2003, en Aguilar, Carvajal, Cerdas, Céspedes, Monge, Ovares, et al. (2002) considera que

... la necesidad de revalorar y contemplar a los habitantes del medio rural, sus familias, sus comunidades, sus relaciones y su concepción de mundo, sus valores y tradiciones, sus juicios y prejuicios entre otros determinantes, para el planteamiento de propuestas orientadas al desarrollo con impacto social, donde la educación se constituye en el instrumento articulador para dar respuesta a los retos presentes y futuros. (p. 41)

La DER, desde sus orígenes, ha asumido el compromiso de formar docentes atendiendo la particularidad de la zona rural, en procura no sólo de un mejoramiento en la calidad educativa, sino, también, de que ésta produzca un impacto en el desarrollo de las comunidades rurales. Según el Plan de Estudios de la DER, el objeto de estudio está constituido por el "conjunto de procesos educativos que se desarrollan en los espacios formales y no formales de la Educación General Básica en contextos rurales, con la finalidad de realizar trabajos académicos prácticos en el proceso de formación, en el que se contempla las relaciones que se generan desde la escuela con las familias y las comunidades" (UNA. DER, 2007, p. 18). Es por ello que, en su quehacer, orienta acciones académicas para la atención de los procesos de práctica profesional que se desarrollan en los contextos rurales.

Para el abordaje de la práctica profesional de la DER es importante visualizar algunos antecedentes que fundamentan ese proceso. La UNA, con la convicción y proyección que la caracterizan, se ha abocado a la búsqueda de las estrategias necesarias que contribuyan a la construcción del conocimiento y a la formación de profesionales, que con su quehacer promuevan la transformación y el bienestar de la sociedad costarricense. Una de estas estrategias es la Práctica Profesional Supervisada (PPS) aprobada en su Segundo Congreso, la cual establece la necesidad de definir las políticas y los lineamientos que reglamenten su aplicación. En cuanto a esto, el Estatuto Orgánico (UNA, 1993) capítulo XVI, artículo 122 da potestad a las unidades académicas para desarrollar un conjunto de actividades académicas afines con su objeto de estudio o su práctica profesional específica. Asimismo, en el capítulo IV (De los estudiantes), artículo 200, sobre los deberes de los y las estudiantes inciso d. enuncia el cumplimiento del ejercicio profesional supervisado dentro del territorio nacional, como parte de su formación universitaria y de su retribución a la sociedad costarricense, de acuerdo con lo estipulado en el reglamento que cada unidad elabore.

Con base en los criterios establecidos por el Consejo Universitario,

la práctica profesional supervisada (PPS) contribuye a consolidar los conocimientos y destrezas adquiridas, así como el desarrollo de valores y actitudes. Es una experiencia que 
realiza el estudiante en un ámbito específico de aplicación de su especialidad disciplinaria o profesional, a su vez, en un espacio de aprendizaje que posibilita la proyección social del estudiante y el establecimiento de relaciones teóricas, metodológicas y técnicas con la realidad. Todo lo cual concurre a la formación integral de cada estudiante. (p. 1)

Desde el quehacer de la DER, los contextos educativos en los que se insertan nuestras y nuestros estudiantes corresponden al ámbito educativo rural, tanto formal como no formal. Esta especificidad, de acuerdo con nuestro propósito de estudio, establece la necesidad de brindar insumos básicos que delimiten, caractericen y profundicen en aspectos inherentes en el proceso de la práctica profesional que se requiere para la comunidad educativa rural.

De acuerdo con lo que establece en Plan de Estudios de la DER (UNA. DER, 2007), "es importante rescatar que los estudiantes y las estudiantes de la DER se incorporarán al aula escolar de forma constante, lo que les dará importantes herramientas para adquirir experiencias personales y académicas, se dan en un curso específico llamado Práctica Profesional en el Contexto Rural" (p. 22).

La práctica profesional de la DER encuentra su sustento teórico en la pedagogía rural, cuyos postulados enuncian el papel protagónico del docente rural, en liderazgo, gestión y proyección a la comunidad, y al visualizar una escuela de puertas abiertas a la comunidad, en la cual se generan procesos articulados en estrecha relación escuela-comunidad, con la clara concepción de una escuela para el desarrollo. En ella, el elemento principal del currículo son los niños, los cuales tienen características y potencialidades particulares que deben ser aprovechadas y mejoradas, a partir de sus vivencias y su contexto.

En la DER, se concibe la práctica profesional, como un espacio de reflexión para el crecimiento personal, social y académico, en el que la interacción entre los diferentes actores (académicos, docentes, estudiantes practicantes, comunidad estudiantil y contexto rural), promuevan el desarrollo de conocimientos, actitudes, destrezas, valores y vocaciones en el propio campo de la acción, para la formación completa del estudiante. Es una experiencia integral de aprendizaje que inserta al practicante en un contexto rural en el que articula la teoría con la práctica mediante su desempeño como docente, y demuestra las competencias básicas para desenvolverse profesionalmente como un maestro o maestra con pertinencia en la escuela rural. Este proceso, en un ámbito rural, es un espacio de oportunidad, tanto para la apropiación teórica y metodológica como para el desarrollo innovador y significativo que construya el o la estudiante en liderazgo y gestión, como requisitos básicos en su perfil profesional para lograr la proyección social (Vargas, 2009). Es un proceso que enfrenta al estudiante con una realidad de aula, en la que tiene que asumir la complejidad del grupo en cuanto a disciplina, planear e impartir las lecciones y desarrollar algunas acciones inherentes a la labor del docente regular y, también, visualizar el contexto rural como entorno del estudiante, el cual debe ser abordado metodológicamente.

El contexto educativo en el que se realiza la práctica profesional, comúnmente, ha sido las escuelas ubicadas en las zonas rurales, las cuales tienen poca matrícula, por lo que son clasificadas como escuelas unidocentes, dirección 1 y dirección 2. También se contemplan algunas escuelas dirección 3 ubicadas en una zona rural concentrada, la cual se caracteriza por estar organizada en dos grandes áreas: una con las viviendas agrupadas y otra con los espacios dedicados al cultivo ${ }^{3}$. En el primer grupo de escuelas, el docente regular atiende a un grupo o varios a la vez, como es característico en las escuelas multigrado.

${ }^{3}$ Según resolución No DCG-097-2009 de la Dirección General de Servicio Civil, del 15 de abril de 2009. Las escuelas unidocentes comprenden una matrícula hasta de 30 estudiantes, D1 de 31 a 90 estudiantes, D2 de 91 a 200 estudiantes y una D3 de 201 a 400 estudiantes. 
Los espacios que se ofrecen para práctica profesional incluyen: escuela diurna, (unidocente, dirección 1 o dirección 2), escuela nocturna, programas de alfabetización y otros servicios que se puedan ofrecer, en los que se ejerza la docencia y sean avalados por la DER y la Comisión de Práctica profesional.

\section{Implementación del Proyecto Intercambio Académico (PIA)}

La DER planteó en 2006, la ejecución de un proyecto para dar respuesta a la falta de cohesión entre el proceso de aplicación de conocimientos construidos por los estudiantes y las escuelas en las cuales desarrollan la práctica profesional. Por tanto, el proyecto se orientó al cultivo de escenarios educativos que brindaran los espacios y las relaciones propicias para el desarrollo de la práctica profesional de la DER. Con igual primacía, el proyecto pretendió la capacitación y la actualización de los maestros y las maestras de las escuelas que colaboran en la formación inicial de los docentes, acción que jugó un papel fundamental en el proceso articulador. El proyecto Intercambio Académico se implementó con dos objetivos generales:

- Crear un intercambio académico entre la División de Educación Rural y las escuelas rurales colaboradoras de la Región Educativa de Heredia, para que el estudiantado, con base en su experiencia, comprenda que los conocimientos se adquieren de manera integrada y articulada con el medio en el que se desarrollan los niños y las niñas.

- Promover la capacitación y la actualización de docentes colaboradores ubicados en las escuelas rurales de la Región Educativa de Heredia.

Para el logro de los objetivos se tomó en cuenta la participación de académicos de la DER, funcionarios de la Dirección Regional de Educación de Heredia, la Comisión de Práctica Profesional, estudiantes del II nivel de Diplomado, docentes de escuelas colaboradoras e invitados expertos en temas abordados.

Para concretar el proyecto y lograr los objetivos, se desarrolló una serie de actividades, las cuales se exponen a continuación.

\section{Divulgación y diagnóstico}

El PIA se inició con un proceso de divulgación mediante el cual se dio a conocer y se desarrollaron los mecanismos para la articulación con escuelas e instituciones involucradas, con la participación y la anuencia de los estudiantes, quienes fueron el primer enlace entre las escuelas y la DER. Esta etapa comenzó en 2006. Paralelamente a la difusión, se ejecutó un diagnóstico para detectar las áreas de capacitación que requerían las escuelas que apoyaban la formación docente de la DER.

Según los datos recabados durante el proceso de investigación diagnóstica se destacaron cuatro áreas prioritarias: planeamiento didáctico, evaluación de los aprendizajes, adecuaciones curriculares y temáticas relacionadas con la matemática. Toda esta información fue suministrada por los directores de las escuelas que recibieron estudiantes practicantes de la DER ese año. Como segundo paso, después de analizar la información obtenida, se establecieron los mecanismos y las relaciones necesarias para responder a las necesidades detectadas por medio del planteamiento de estrategias para la vinculación de instituciones y profesionales al proyecto. 
La propuesta se llevó a cabo mediante el enlace y la articulación de PIA-DER con la Dirección Regional Educativa de Heredia; para ello se contó con el visto bueno de la Directora Virginia Jara y de los jefes del Departamento de Desarrollo Educativo 2007-2008, elemento clave para lograr la asistencia y la concentración de los docentes invitados, ya que desde esa instancia se tramitó la convocatoria a los talleres. Durante 2007, se contó con la valiosa participación de las funcionarias de la Asesoría de Educación especial, Asesoría de evaluación, Asesoría en capacitación y Desarrollo profesional de la Dirección Regional de Heredia. En el 2008, se contó con la participación activa de la Asesoría de Español a cargo de la M. ED. Sandra Chacón.

Se rescata que, mediante el trabajo conjunto UNA-MEP, se buscó solventar las necesidades de capacitación de docentes, con lo que se demostró el compromiso y la responsabilidad de funcionarios e instituciones con injerencia directa en la formación de profesionales en el campo de la docencia.

\section{Plan de capacitación}

Con base en las áreas diagnosticadas se establecieron ocho temáticas para desarrollar en talleres, con la finalidad de fortalecer la práctica pedagógica en el aula escolar mediante el plan de capacitación y de actualización, dirigido a los y las docentes de escuelas colaboradoras en el marco del PIA, de la DER.

El proceso de capacitación se inició con tres talleres de actualización en materia de adecuación curricular, evaluación de los aprendizajes y planeamiento didáctico. En estos talleres, participó un promedio de 22 educadores. Posteriormente, se plantearon dos etapas para la capacitación y la actualización de docentes en servicio, para lo cual se desarrolló una serie de talleres teóricoprácticos de aprovechamiento, con certificación de cada una de las etapas. En la primera etapa se realizaron ocho talleres dirigidos a docentes de I ciclo y en la segunda etapa se desarrollan seis talleres destinados a docentes de II ciclo.

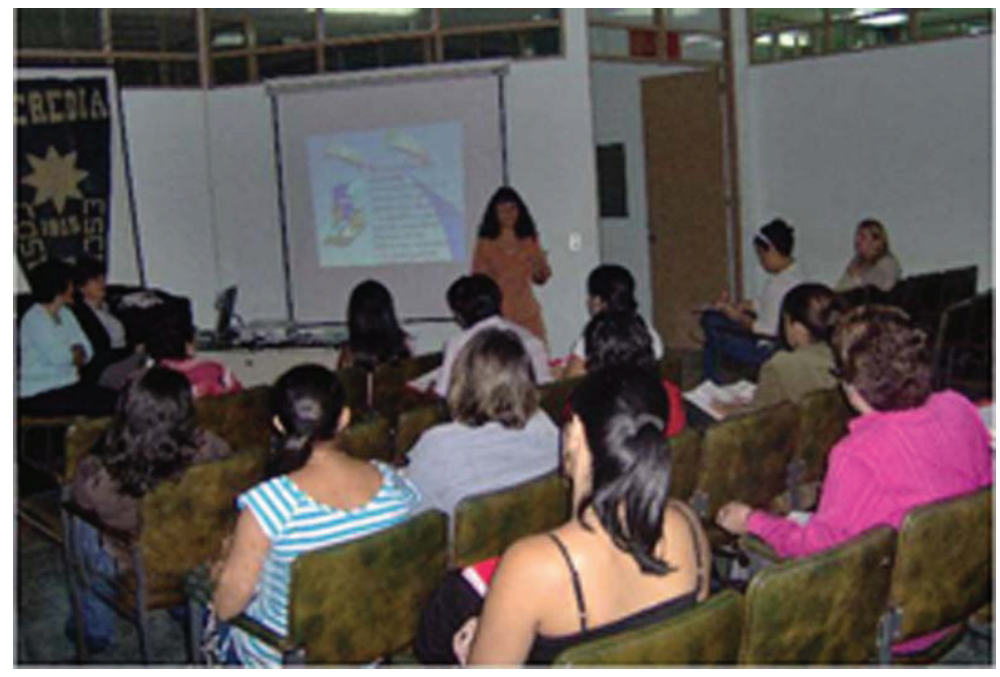

Figura 1. Taller en coordinación con la Asesoría de Educación especial, 2007.

\section{Contacto directo con la realidad educativa rural}

En el desarrollo del PIA se contempló la realización del proceso de práctica profesional, para lo cual se tomaron en cuenta las posibilidades laborales y espacio-temporales de los estudiantes, y la actividad fue valorada como práctica profesional distribuida o intensiva. La práctica profesional distribuida está dosificada en 12 horas semanales, repartidas a lo largo del calendario escolar; en 
ella se contemplan los meses de agosto a noviembre. La práctica profesional intensiva ofrece la oportunidad para que el estudiante realice una labor sin interrupción en el aula escolar; esta última opción de ejecución fue avalada por la Comisión de Práctica profesional, para ayudar a aquellos estudiantes que experimentaban situaciones justificadas para optar por esta modalidad.

El desarrollo de la práctica docente se llevó a cabo en varias etapas. En la primera etapa, se dio un proceso de inducción para motivar, para aclarar dudas y reducir el nivel de ansiedad de los estudiantes. En la segunda etapa, se establecieron los espacios y los mecanismos que permitieron orientar, oportunamente, al estudiante en cuanto a requisitos, responsabilidades y restricciones durante el proceso de práctica. En la tercera etapa, se insertó al estudiante en el aula escolar con la orientación del curso teórico y el apoyo de un profesor tutor, el cual fungió como un guía que orientó y dio seguimiento a los avances de los estudiantes.

La supervisión de la práctica implicó el desarrollo paralelo de un proceso de acompañamiento, el cual requirió la interacción armoniosa de dos sujetos del proceso: el profesor tutor de la práctica (evaluador) y el estudiante-practicante (evaluado). El profesor tutor tuvo la responsabilidad primordial de atender al alumno practicante, para que éste efectuara una labor docente con un mayor nivel de seguridad, y mantener la comunicación directa con él y con los maestros colaboradores. El acompañamiento pedagógico, como proceso de supervisión, fue la mejor evidencia observable y valorable del carácter profesional, sobre el desempeño del estudiante en una escuela rural.

La práctica profesional tiene una duración promedio de cuatro meses. En ella, el o la estudiante tiene la oportunidad de confrontar la teoría con la práctica, al insertarse en un contexto educativo rural. Durante esta etapa, el o la estudiante tuvo la responsabilidad de cumplir con un horario establecido, elaborar y ejecutar un planeamiento didáctico para demostrar, en la práctica, conocimiento didáctico, apropiación metodológica, manejo de grupo e interacción armoniosa con los demás actores de la comunidad educativa, $y$, todavía, trascender el espacio áulico mediante la realización de un taller de extensión dirigido a la comunidad educativa.

En la cuarta y última etapa de la práctica profesional, los y las estudiantes practicantes, los docentes colaboradores y los académicos involucrados valoraron diversos aspectos, entre ellos: la inducción y el acompañamiento durante el proceso de práctica, las dificultades que se presentaron durante la ejecución de la práctica docente, las estrategias que se utilizaron para enfrentar los obstáculos, así como las conclusiones y las recomendaciones del proceso realizado. Los resultados de las valoraciones fueron muy positivos, tanto de la organización del proceso como del acompañamiento que recibieron de los académicos.

Las mayores dificultades que se evidenciaron fueron la suspensión de clases en las escuelas y la discrepancia entre la metodología del docente regular y la propuesta por el estudiante practicante.

\section{Productos}

Los mayores logros obtenidos a través del proceso fueron los siguientes:

- La capacidad social construida. Se capacitó a 73 docentes en servicio, con un 121,66\% del producto logrado, pues se superó lo establecido: capacitar a 60 docentes. Se involucraron los circuitos educativos 02, 03, 04 y 05 de la Dirección Regional de Heredia, con un total de 31 escuelas que recibieron los talleres de capacitación y de actualización. Se realizaron 21 talleres: tres dirigidos al proceso de inducción de los estudiantes al proceso de práctica profesional y 18 orientados a docentes de I y II ciclos de la Educación General Básica. 
Además, 15 talleres desarrollados por estudiantes como propuesta de extensión a las comunidades educativas rurales.

- Creación de material didáctico y pedagógico. Se intentó contar con una propuesta para establecer los lineamientos generales de la práctica profesional supervisada. Como producto se obtiene, no sólo la creación de un documento con lineamientos normativos, sino que se crea, también, una guía orientadora del proceso y se ofrece un compendio de instrumentos creados, igualmente, para registrar, organizar y sistematizar el proceso de práctica profesional.

\section{A modo de conclusión}

- Existen grandes retos para la DER y para quienes asuman el proceso de práctica profesional, ya que una realidad con la que hay trabajar, es el ingreso de estudiantes que escogieron la carrera como única alternativa para el acceso a la universidad y, además, que tienen una visión completamente citadina. Si bien esto es una debilidad, se convierte, a la vez, en un reto: que el estudiante conozca las bondades afectivas de la profesión docente por desarrollar en una zona rural, las grandes alegrías de ser instrumento para el aprendizaje de otros, con limitaciones materiales, contextuales y referentes al acceso de las nuevas tecnologías, pero con gran potencial y riqueza natural, que están a la espera de unos ojos nuevos, creativos, sensibles, que fijen su mirada en un bello porvenir para la escuela y la comunidad y que, con base en ello, lidere y gestione procesos de desarrollo escolar y comunal.

- En la formación profesional, desde la especificidad de la educación rural, se deben reforzar y promover acciones con la intención de que los estudiantes:

- interioricen que las comunidades del país son heterogéneas, que tienen una cultura cotidiana diferente, su forma de visualizar la vida, sus percepciones, su concepción de mundo, expectativas, anhelos, intereses y necesidades son particulares y que la propuesta educativa que se plantee valore y articule los saberes locales, para lograr la significatividad en los procesos de enseñanza y de aprendizaje, y desde ella se respete y se potencie el amor y el arraigo hacia su contexto, se aprovechen los recursos de la comunidad y se articulen los diferentes procesos de desarrollo con la escuela. El desempeño de los estudiantes debe ir mucho más allá, para que reconozcan y asuman las posibilidades y carencias del entorno escolar, que no se encierren en las cuatro paredes $\mathrm{y}$, mucho menos, que consideren que su función termina después de los límites de propiedad de la escuela; por el contrario, se visualiza una escuela de puertas abiertas a la comunidad, y que la escuela y la comunidad constituyan un binomio, desde el cual se promueva el desarrollo local.

- construyan una formación sólida en actitudes y valores, para dar autenticidad a los estudiantes formados en la DER, y que no sucumban ante el tradicionalismo ni se dejen absorber por un sistema enraizado en el que existe primacía de la burocracia ante lo pedagógico, el círculo vicioso de un sistema de dejar pasar, dejar hacer; con criterio para debatir, luchar por los ideales no tanto propios, sino de los niños que estén bajo su tutela; tener el valor para sobrepasar el sistema cuando las circunstancias lo ameritan, y que visualicen al niño y la niña como seres con necesidades e intereses individuales, sujetos de derecho y que aboguen por el bien común, asumiendo la profesión con compromiso y con pasión. 
- En los cursos que preceden a la práctica profesional se necesitan mayores propuestas de campo, para que los estudiantes tengan contacto directo y permanente con la realidad rural, y que estos acercamientos, con actitud crítica, les provea diversas realidades contextuales, diferencias metodológicas, procesos cotidianos que se realizan en las escuelas y que forman parte del diario vivir del docente (trabajo administrativo, celebraciones patrias, patronales y religiosas; campañas estudiantiles, en salud, de bien social, entre otras) . El poco contacto que tienen cuando ingresan al proceso repercute en que, muchas veces, su inserción en la escuela, como estudiante practicante, la perciban, en algunos casos, un poco abrupta. La inserción de un estudiante debe ser el resultado de un proceso secuencial y articulado, con acciones de base que solidifiquen la autoconfianza como docente y que brinden insumos para su fortalecimiento, para que cuando asuman un grupo escolar lo hagan con propiedad y el mayor empoderamiento posible.

- A partir del desarrollo del proyecto se pueden establecer varios beneficios: se logró mejorar la organización del proceso de práctica profesional, así como establecer un mayor contacto y una vinculación de la DER con los docentes y directores de escuelas rurales; a la vez que se realizaron acciones de retribución a las escuelas colaboradoras mediante la ejecución del plan de capacitación. En términos de vinculación, se puso en contacto la UNA con diferentes contextos escolares de nuestro país, se generó un acercamiento de docentes en servicio a esta casa de enseñanza y se consolidaron escenarios educativos rurales para el desarrollo de futuras propuestas. En este proceso, se puso de manifiesto que la formación que reciben nuestros estudiantes regulares se circunscribe a un tiempo histórico-social determinado y que los docentes en servicio, en esta época, deben enfrentar una realidad educativa muy diferente de cuando recibieron su proceso de formación docente, lo cual es un insumo valioso por considerar en las propuestas pedagógicas y curriculares que ofrece la universidad.

- Es rescatable el alto grado de vinculación que se logró con el proyecto, lo que dio como fruto la consolidación de un grupo de escuelas colaboradoras de la Dirección Regional de Heredia, como apoyo permanente en los procesos de práctica profesional de la DER.

- El proceso de práctica profesional es una estrategia comunicativa y de enlace idóneo para desarrollar acciones de investigación y de extensión en comunidades educativas rurales.

- Se debe rescatar la gran capacidad de convocatoria que tiene la UNA, el prestigio que la caracteriza, lo cual contribuyó en la creación de alianzas, en la anuencia y permanencia de las instituciones y de las personas que participaron en el desarrollo del plan de capacitación.

- Es importante visualizar algunas percepciones de los participantes en el plan de capacitación, que dan muestra del impacto producido:

- Los docentes en servicio que participan en el plan de capacitación reconocen vacíos, carencias y limitaciones, para enfrentar el quehacer cotidiano en el aula; y conciben la actualización continua de los maestros como una necesidad prioritaria. Esto se ilustra con el comentario de un docente quien afirmó que "todos los talleres recuerdan al educador, que el ser maestra o maestro es una vocación y que, por ética profesional, deben estarse actualizando"; o bien; el comentario de una maestra que expone: "para mí, en particular, esta capacitación fue de gran valor, ya que aprendí gran cantidad de actividades que hoy por hoy las pongo en práctica con mis alumnos, a pesar de tener la edad que tengo siempre he dicho que nunca es tarde para actualizarse, (....) quisiera pedir ser tomada en cuenta en próximas capacitaciones como éstas, que son de suma importancia en el quehacer educativo". 


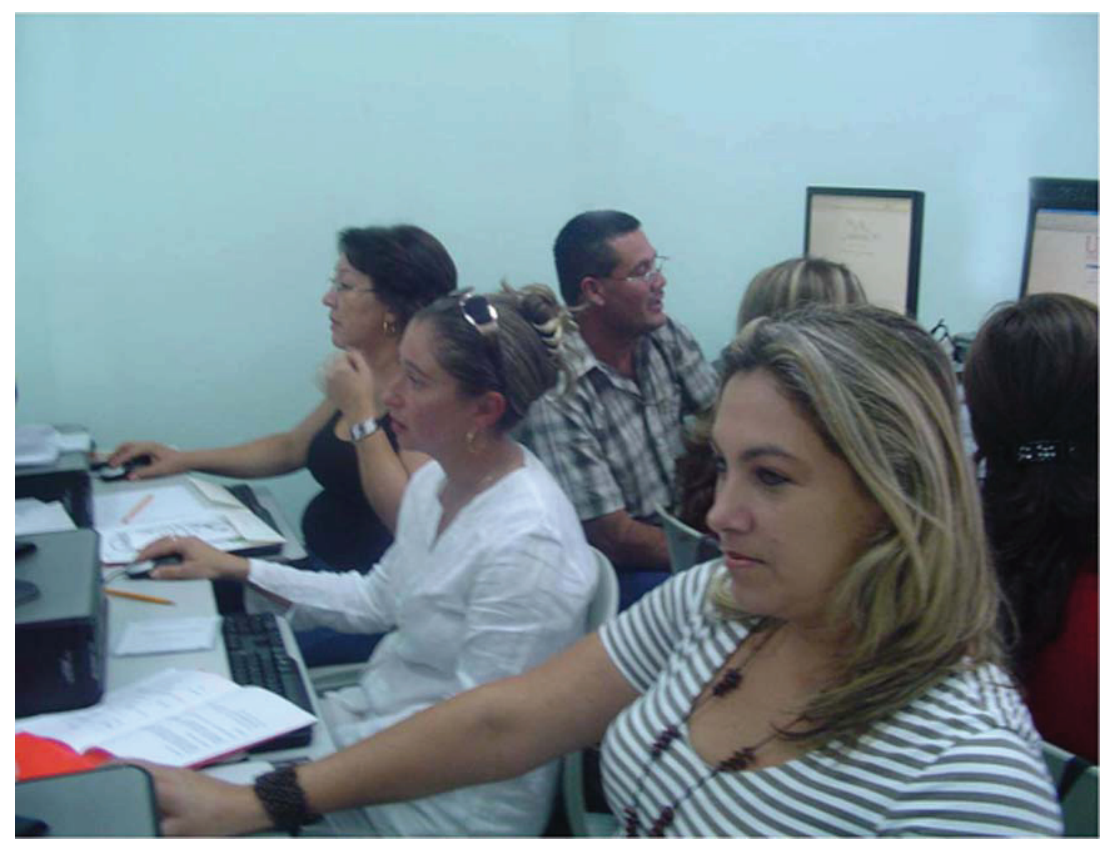

Figura 2. Docentes que participaron en la II etapa del Plan de Capacitación, 2008.

- El proyecto promovió ese intercambio académico, ese compartir entre colegas, para exponer sus vivencias, confrontar sus realidades, plantear opciones. El comentario de una docente participante lo ilustra: "quizás se considera que lo más importante de esta formación ha sido el refrescar conocimientos en diferentes ámbitos, pero creo que, además, hemos podido compartir con otros profesionales, experiencias diarias que no están escritas en ningún libro...”.

- El proyecto evidencia que el brindar espacios para la capacitación y la actualización con temática pertinente, aplicable y promovida en procesos significativos, ofrece oportunidades que son bien valoradas y aprovechadas por parte de los y las docentes. Al respecto, una docente expresó: "en siete años de mi labor docente no me había interesado tanto en una capacitación. Fue tan motivante que esperaba con ansias el día de la capacitación para ver lo que se iba a aprender y practicar ese día”, y esto fue reforzado con el siguiente comentario: "como docente de educación primaria me siento muy satisfecha de haber tenido la oportunidad de crecer y mejorar la calidad de mi trabajo; gracias a estos talleres muchos docentes podemos ver la educación desde otra perspectiva y, a la vez, motivar a otros docentes a enriquecer sus conocimientos e interesarse por recibir capacitaciones...”.

- Uno de los aspectos más significativos durante el desarrollo de los talleres de capacitación, fueron los datos aportados en torno a la percepción de cada actividad y el nivel de apreciación que, voluntariamente, los participantes brindaron, con expresiones cualitativas con reiterado agradecimiento, impregnadas de emotividad, cariño y espiritualidad; ejemplos de ello: "Gracias por la experiencia, anécdotas y estrategias compartidas durante todo este tiempo. Gracias porque aún se puede creer en las personas que se preocupan por ayudar al docente a facilitar su labor cotidiana...” o “...que Dios les bendiga y les siga concediendo ese espíritu de lucha por mejorar la labor pedagógica docente, y brindar oportunidades para una mejor calidad de vida personal y ciudadana...”. 


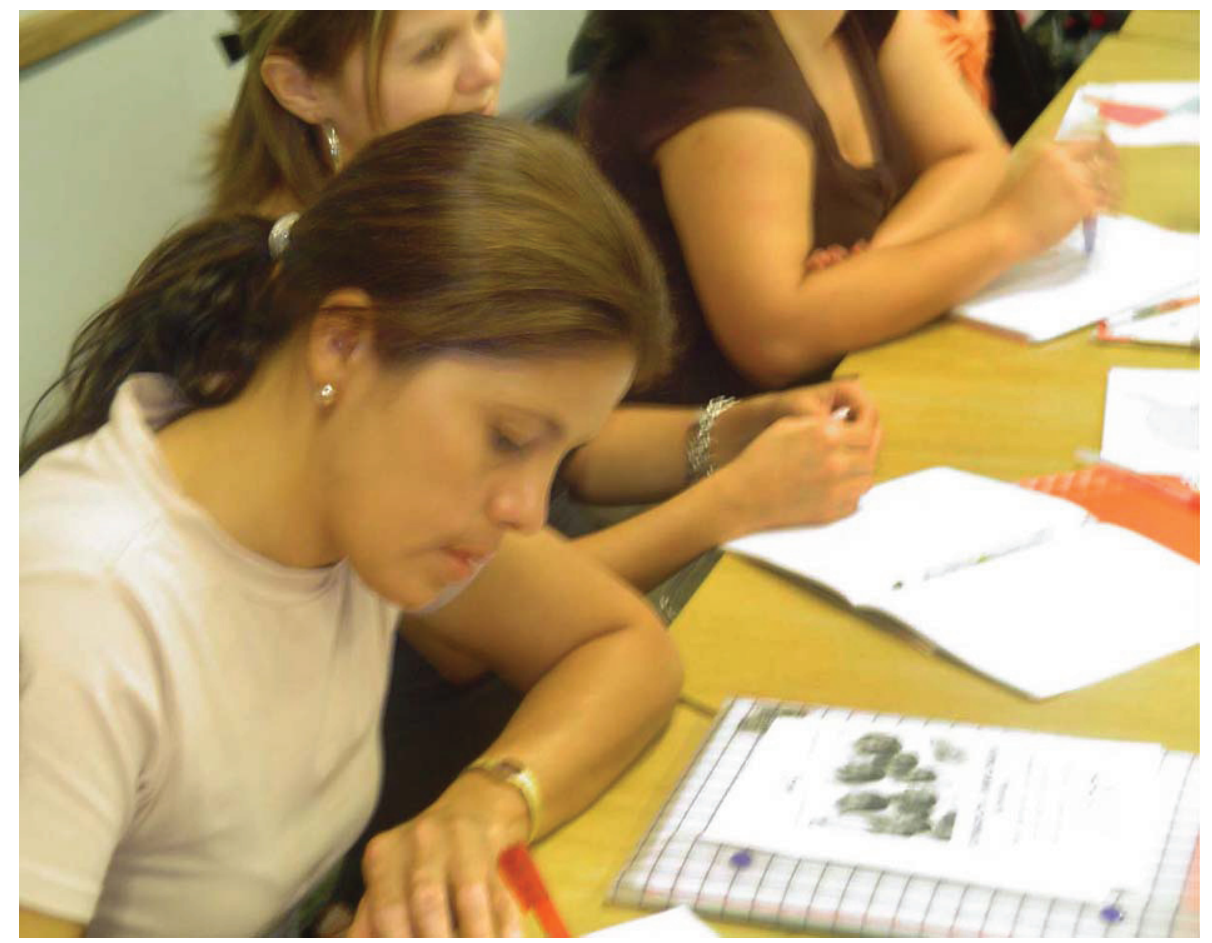

Figura 3. Taller de lectoescritura en el que participaron los docentes colaboradores.

\section{Recomendaciones a partir de las lecciones aprendidas}

- La práctica profesional es una actividad académica que no debe realizarse en forma aislada y sin conexión con otros procesos, por lo que es importante que se promueva, como actividad continua, en la unidad académica. Esta propuesta permite: a) el análisis permanente y profundo para evidenciar las fortalezas y las debilidades de la práctica profesional; b) con base en ello, plantear las mejoras que orienten y nutran los procesos que se promueven; c) detectar nichos educativos rurales de calidad que sirvan como modeladores a los estudiantes practicantes, para que ellos crean que es posible hacer las cosas de manera diferente (que se puede innovar y crear desde una estructura estatal) y d) identificar situaciones desfavorables que vivencian docentes y estudiantes, y a las cuales se pueda dar respuesta desde algunas propuestas de la DER.

- Es importante rescatar que, en los diferentes procesos académicos que se desarrollan en la educación superior, los profesionales son formados con poco o ningún insumo sobre una concepción de la ruralidad, en la que se homogeniza las relaciones, los contextos y, sobre todo, los procesos de práctica profesional. En las diferentes carreras o profesiones que se ofertan en las universidades, es común que se les proponga a los estudiantes un trabajo de campo o, bien, una práctica profesional, en la que apliquen y demuestren los conocimientos requeridos en su profesión. Las diferentes actividades profesionales de campo que se plantean (tanto cognitivas como operatorias básicas como perfil de salida de cada profesional), van de acuerdo con la carrera, su objeto de estudio, los conocimientos, las destrezas y las habilidades. Pero es relevante que, en cada caso de formación profesional, se asuma un trabajo de campo o práctica profesional específica y no con un concepto general. En el caso de la DER, debe preparar a los profesionales para que sepan enfrentar y asumir diferentes 
realidades: lo urbano, lo rural, lo marginal, lo rural marginal, ya que no sólo deben atender particularidades pedagógicas, administrativas y técnicas, sino también contextuales, las cuales influyen en gran medida, y que requieren un tratamiento metodológico apropiado, como fruto del conocimiento promovido por la universidad. Por consiguiente, es importante preparar a los profesionales para que conozcan diferentes escenarios dentro de los cuales pueden incursionar, para una adecuada intervención profesional. Hay que reconocer que la mayoría del territorio nacional es rural.

- Es necesario realizar evaluaciones de contexto sobre la realidad rural y el proceso de práctica profesional, en el que se valore el aporte de los diferentes actores, sean partícipes directos o indirectos, interesados, destinatarios o desarrolladores de los procesos, entre muchos otros. Asimismo, deben analizarse las relaciones que se generan en estos contextos, ya que el problema de la desventaja educativa, social y económica que enfrentan las zonas rurales es multifactorial y pluridimensional, y para dar una respuesta asertiva a éstos, debe hacerse una lectura de su realidad para crear propuestas emergentes, articuladas y participativas, si se quiere hacer aportes significativos y con impacto global.

- Es importante aprovechar las lecciones aprendidas, las estrategias logísticas, los vínculos y los productos generados en el PIA, los cuales pueden contribuir a enriquecer y a mejorar las propuestas educativas para la formulación de futuros proyectos. Con ello, se puede asumir el compromiso de fortalecer los procesos de educación continua y los procesos de práctica profesional, pues los proyectos constituyen un medio eficaz para realizar acciones de impacto educativo y social.

\section{Referencias bibliográficas}

Aguilar, M. (1994). Hacia una pedagogía rural. Heredia, Costa Rica: Universidad Nacional.

Aguilar, M., Carvajal, N., Cerdas, Y., Céspedes, E., Monge, M., Ovares, S. et al. (2002). Educación rural: un acercamiento pedagógico. Heredia, Costa Rica: Universidad Nacional.

Gajardo, M. y Andraca, A. M. de (1992). Docentes y docencia en zonas rurales. Santiago, Chile: UNESCO-FLACSO.

Universidad Nacional. División de Educación Rural. (2007). Plan de Estudios. Heredia, Costa Rica: Comisión Curricular.

Universidad Nacional. (1993). Estatuto Orgánico de la Universidad Nacional. Heredia, Costa Rica: Autor.

Vargas, M. (2009). Guía Orientadora de la Práctica Profesional de la División de Educación Rural. Heredia, Costa Rica: Universidad Nacional.

\section{Otra fuente consultada}

Solano, J., Kampen, P. y Ovares, S. (2003). El docente rural en Costa Rica: radiografía de una profesión. Heredia, Costa Rica: Editorial Universidad Nacional. 